\title{
INCIDENCIA DE LOS FACTORES PSICOSOCIALES EN EL TRABAJO. UN ESTUDIO EN DOCENTES UNIVERSITARIOS
}

Ortega Domínguez, S.; Sánchez Díaz, C.

\section{RESUMEN}

El presente estudio se adentra en las características de la relación existente entre las condiciones del trabajo y su organización, y la salud de las personas. Este es el punto de partida para analizar y discutir mediante el método Istas21, la incidencia de los factores psicosociales relacionados con la organización del trabajo y sus consecuencias en la salud de los docentes de un centro universitario.

Palabras clave: factores psicosociales, organización del trabajo, trabajo, estrés, condiciones del trabajo.

\section{INTRODUCCIÓN}

El trabajo es una actividad dentro de la realidad social en la que vivimos, y como tal puede tener una repercusión sustancial en el bienestar o malestar en las personas. Estas repercusiones en el estado afectivo de los trabajadores, no implican la existencia de características inherentes al trabajo que nos definan este tipo de situaciones, sino que son los factores psicosociales que rodean la organización del trabajo los que inciden en estas consecuencias de bienestar o malestar. (Córdoba, 2008).

\section{Los factores psicosociales y el estrés laboral}

Podemos entrever una serie de características en las condiciones del trabajo y en su organización, que afectan a la salud de las personas (ISTAS, 2002) y que les expone a una serie de riesgos de origen laboral (Luceño y otros, 2005). Estos riesgos de origen laboral se manifiestan a través de una serie de mecanismos psicológicos y fisiológicos donde se identifica al estrés como precursor de la enfermedad. De acuerdo con esta orientación, el estrés como proceso psicológico es la base de la relación entre la organización del trabajo y la salud de los empleados. Delimitamos entonces esta relación entre factores psicosociales y salud como una fuente de estrés 0 de reacciones de índole emocional, cognitiva, fisiológica o del comportamiento a ciertos aspectos adversos de la organización o del entorno de trabajo (Comisión Europea, 1999), que generan una sensación de frustración, al no poder hacer frente la situación.

\section{Incidencia del problema}

La salud laboral de los trabajadores en los centros docentes públicos es un problema al que, desde hace varios años, se enfrenta la Administración pública. Estudios realizados por comisiones obreras (2006) en la provincia de Málaga nos muestra que trabajar como docente 
expone a una serie de riesgos, que según Rabada (2006), se relaciona con una serie de trastornos en la salud.

Los estudios, centrados en los problemas de salud causados por el trabajo en las enseñanzas no universitarias, no nos arrojaban datos válidos para observar la incidencia de los factores psicosociales en la salud de los docentes de centros universitarios, presentándose el objetivo de evaluar la incidencia de una serie de factores en la salud de los docentes de nuestra propia universidad.

\section{Evaluación de los riesgos psicosociales}

La importancia de la evaluación de riesgos psicosociales en la salud de los docentes, radica en servir de base para gestionar de manera activa la seguridad y la salud en el trabajo, pudiéndose evitar desde los centros que las situaciones del trabajo puedan ser causa de una enfermedad en sus trabajadores. (Ley 31/1995, de 8 de noviembre, de Prevención de Riesgos Laborales).

A diferencia de lo que ocurre con parte de los riesgos físicos y biológicos, que pueden ser evaluados mediante medidas fiables y válidas para evaluar la exposición al riesgo (Luceño y otros), los riesgos psicosociales en este estudio han sido evaluados de forma subjetiva mediante la colaboración de diez docentes de uno de los departamentos de una facultad de la Universidad de Granada. La dificultad de medir de forma objetiva las características del trabajo que se definen en el método Istas21, radica en la necesidad de evaluar estos factores teniendo en cuenta la percepción del trabajador sobre las mismas, es decir, mediante la medida subjetiva de los factores sobre las condiciones de trabajo y su organización.

Entendemos que identificar el nivel de exposición psicosocial más o menor favorable para la salud a la salud a la que están expuestos los trabajadores mediante las diversas cuestiones sobre dimensiones psicosociales del método ISTAS21, constituye el primer paso para poder intervenir y tomar medidas orientadas a su prevención. La evaluación de los factores psicosociales de riesgo y los problemas de salud en los trabajadores cobra una gran importancia en los centros educativos y nos invita, a través de este estudio, a contribuir a la discusión y el análisis acerca de los factores psicosociales en la organización del trabajo en un centro universitario, y sus consecuencias para la salud de los docentes.

\section{MÉTODO}

\section{Muestra}

Los diez docentes participantes en el estudio pertenecen a un departamento con menos de 25 trabajadores de un centro universitario. La selección de la muestra se ha realizado en función de la disponibilidad de los/las docentes, por lo que los resultados de este estudio no son representativos de la población ni pueden ser generalizables. La muestra consta de un número equiparado de hombres y mujeres, en su mayoría profesores titulares, ayudantes o sustitutos de Universidad. 


\section{Instrumento}

El método ISTAS21 es una adaptación para la población española del Cuestionario Psicosocial de Copenhagen (CoPsoQ). Este instrumento fue seleccionado como la principal herramienta para la recogida de datos por su carácter individual, anónimo, confidencial y de respuesta voluntaria. Estos datos nos permitieron realizar un estudio cualitativo de la realidad objeto de evaluación en una población específica, en este caso docentes de centros universitarios como una medida de prevención orientada a favorecer la localización de determinados problemas y vías alternativas de cambio o mejora, con el fin de impulsar otras formas de organización laboral más saludables.

El método Istas21, versión corta, es un cuestionario de cuatro secciones, dos de ellas orientadas a identificar las condiciones sociales y de empleo, y las otras dos centradas en determinar aquellos daños o dimensiones psicosociales que tengan algún efecto sobre la salud. De manera más específica, en el test se analizan seis apartados o dimensiones psicosociales: (1) exigencias psicológicas, (2) trabajo activo y posibilidades de desarrollo, (3) inseguridad, (4) apoyo social y calidad de liderazgo, (5) doble presencia y (6) estima. Definimos a continuación cada uno de estas dimensiones (Istas21, 2002) a evaluar.

De las exigencias psicosociales se distingue por un lado, las de tipo cuantitativo referidas al volumen de trabajo solicitado respecto a la falta de tiempo para ejecutarlo adecuadamente; $y$ las exigencias cualitativas, determinado por las demandas emocionales (al tener que reprimir sentimientos y opiniones), cognitivas (precisando grandes esfuerzos intelectuales) y de tipo sensorial.

En cuanto al trabajo activo y las posibilidades de desarrollo, indica la influencia y control sobre el trabajo a realizar, su organización, horarios, decisiones y mejoras; que posibilitan la integración en la empresa y dan sentido al trabajo. Las posibilidades de desarrollo en el trabajo no deben verse sólo como una oportunidad para ascender, sino para generar expectativas de superación y progreso.

El Apoyo social hace referencia al tipo de relaciones y ayuda entre compañeros, es decir, el sentimiento de grupo y los refuerzos, que requieren de un buen flujo de información que evite el aislamiento y posibilite las relaciones sociales. La calidad de liderazgo entiende que se debe mantener bien definidos los puestos de trabajo (claridad de rol), no exigiendo tareas contrarias a los valores personales (conflicto de rol) y proporcionando la información necesaria para adaptarse a los cambios (previsibilidad).

La inseguridad laboral incide en aquellos aspectos relacionados con la estabilidad laboras, la posibilidad de un cambio de tareas o en relación a una posible promoción y los cambios imprevistos que perjudiquen al trabajador (turnos, días de la semana...).

La estima por su parte pone de relieve el reconocimiento al trabajo y el esfuerzo, recibiendo un adecuado apoyo social y un trato justo.

La doble presencia hace referencia a la incapacidad de compatibilizar las tareas familiares y laborales, aun cuando existe una normativa para la conciliación de la vida laboral y familiar. 


\section{Procedimiento}

Tras la presentación individual del objeto de estudio y el método a utilizar, en este caso el cuestionario Istas21, se pidió la colaboración de los docentes de un mismo departamento universitario. En el momento de la recogida del cuestionario, se solicitó una valoración personal que pudiera ayudar a la interpretación de los resultados del test, conduciéndonos las reflexiones del profesorado a un mejor análisis de los resultados.

\section{ANÁLISIS DE DATOS}

En el departamento evaluado han participado 10 docentes, de los cuales un profesor colaborador tres profesores titulares de universidad, un profesor sustituto interino, un catedráticos de universidad, un profesor ayudante doctor y dos profesores contratados doctor y un profesor asociado laboral, por lo que entendemos que el desarrollo de su funciones varía en relación al contrato al que están sujetos.

En la tabla que mostramos a continuación mostramos los grupos de dimensiones evaluador en el cuestionario "versión corta" Istas21 en relación al nivel de exposición de los docentes, remarcando aquellas cuestiones básicas que han tenido mayor incidencia en sus respuestas.

\section{Tabla 1. Datos obtenidos del cuestionario Istas 21}

\begin{tabular}{|c|c|c|}
\hline DIMENSIÓN & NIVEL DE EXPOSICIÓN & SITUACIÓN \\
\hline $\begin{array}{l}\text { Exigencias } \\
\text { Psicológicas }\end{array}$ & $\begin{array}{l}\text { Nivel rojo: } 80 \% \text { nivel de exposición psicosocial } \\
\text { más desfavorable. } \\
\text { Nivel amarillo: } 20 \% \text { nivel de exposición } \\
\text { psicosocial intermedio }\end{array}$ & $\begin{array}{l}\text { "Siempre" o "muchas veces" existen } \\
\text { dificultades para olvidar los problemas del } \\
\text { trabajo. } \\
\text { "Muchas veces" el trabajo, en general, es } \\
\text { desgastador emocionalmente. }\end{array}$ \\
\hline $\begin{array}{l}\text { Trabajo activo y posibilidades } \\
\text { de desarrollo }\end{array}$ & $\begin{array}{l}\text { Nivel verde: } 80 \% \text { nivel de exposición } \\
\text { psicosocial más favorable para la salud. } \\
\text { Nivel rojo: } 10 \% \text { nivel de exposición psicosocial } \\
\text { más desfavorable. }\end{array}$ & $\begin{array}{l}\text { "Siempre o casi siempre": El trabajo } \\
\text { requiere iniciativa } \\
\text { Permite aprender cosas nuevas } \\
\text { Compromiso con la profesión }\end{array}$ \\
\hline Inseguridad & $\begin{array}{l}\text { Nivel rojo: } 50 \% \text { nivel de exposición psicosocial } \\
\text { más desfavorable. } \\
\text { Nivel amarillo: } 40 \% \text { nivel de exposición } \\
\text { psicosocial intermedio }\end{array}$ & $\begin{array}{l}\text { "Muy preocupado por": Inseguridad por lo } \\
\text { difícil de encontrar trabajo en caso de } \\
\text { perderlo } \\
\text { Inseguridad por el salario } \\
\text { "Bastante preocupado por": Inseguridad } \\
\text { por el salario y el cambios de horario }\end{array}$ \\
\hline $\begin{array}{l}\text { Apoyo social y calidad de } \\
\text { liderazgo }\end{array}$ & $\begin{array}{l}\text { Nivel rojo: } 40 \% \text { nivel de exposición psicosocial } \\
\text { más favorable para la salud. } \\
\text { Nivel amarillo: } 30 \% \text { nivel de exposición } \\
\text { psicosocial intermedio }\end{array}$ & $\begin{array}{l}\text { Puntuaciones bajas en: Planificación del } \\
\text { trabajo por los jefes y la comunicación con } \\
\text { los trabajadores. }\end{array}$ \\
\hline
\end{tabular}




\begin{tabular}{|c|c|c|}
\hline Doble presencia & $\begin{array}{l}\text { No doble presencia: } 20 \% \\
\text { Nivel rojo: } 50 \% \text { nivel de exposición psicosocial } \\
\text { más desfavorable }\end{array}$ & $\begin{array}{l}\text { Realización de la mitad de las tareas en } \\
\text { casa. } \\
\text { Si falta algún día en casa, las tareas } \\
\text { "muchas veces" se quedan sin hacer. }\end{array}$ \\
\hline Estima & $\begin{array}{l}\text { Nivel rojo: } 50 \% \text { nivel de exposición psicosocial } \\
\text { más desfavorable. } \\
\text { Nivel amarillo: } 40 \% \text { nivel de exposición } \\
\text { psicosocial intermedio }\end{array}$ & $\begin{array}{l}\text { Mis superiores me dan el reconocimiento } \\
\text { que me merezco (solo alguna vez) } \\
\text { "Muchas veces" reciben el apoyo } \\
\text { necesario en situaciones difíciles } \\
\text { "Muchas veces" el reconocimiento en } \\
\text { relación al trabajo y esfuerzo es adecuado }\end{array}$ \\
\hline
\end{tabular}

\section{Discusión}

Sobre las exigencias psicosociales, los datos reflejan que existen altos niveles de exposición al riesgo por factores cualitativos, principalmente respecto a las demandas emocionales. Entendemos que estas demandas emocionales están estrechamente relacionadas con los resultados favorables para la salud (niveles altos, $80 \%$ ) en relación al compromiso ante el trabajo como docentes en la dimensión trabajo activo y posibilidades de desarrollo. Es decir, los docentes tienen dificultades para evitar olvidarse de los problemas del trabajo y entienden que es desgastador emocionalmente, lo que se relaciona con un alto nivel de compromiso con el trabajo, precisando de una gran iniciativa en la mayoría de los casos. En esta misma dimensión se observa la poca influencia de los docentes sobre el volumen de trabajo asignado, en ocasiones según los mismos docentes por tener que cubrir bajas o asignaturas no contempladas inicialmente en la planificación docente, y en otras muchas ocasiones, por el poco control en la asignación anual de investigaciones a al profesorado.

El Apoyo social entre los compañeros de departamento es, según sus impresiones, positivo en pequeño grupo, donde creen que siempre existe algún "colega" cercano que les apoyo en el trabajo. Pero generalmente el apoyo social a nivel de departamento es mejorable, y "muchas veces" el profesorado entiende su alto compromiso y esfuerzo no es reconocido por sus jefes inmediatos. Uno de los aspectos a remarcar sobre la calidad de liderazgo, es la poca previsión de los jefes del departamento o del mismo rectorado, para cubrir bajas o sustituir de manera inmediata una materia, sin proporcionarles la información necesaria (previsibilidad) para adaptarse al cambio y ofrecer al alumnado una educación de calidad.

Respecto a la inseguridad laboral, aunque el $60 \%$ del profesorado tiene un contrato laboral fijo, se observa la incertidumbre en relación a la estabilidad laboral. En otras ocasiones también se encuentran preocupados por la reestructuración académica que desde hace varios años se están produciendo derivados del "Plan Bolonia", y más aún, con la desaparición en este curso académico de determinadas licenciaturas relacionadas con este departamento, con los cambios que eso conlleva a nivel laboral.

La percepción de los trabajadores sobre la estima, pone de relieve el poco reconocimiento que conlleva su esfuerzo y trabajo por parte de los jefes inmediatos, pero que según algunos de ellos, es recompensado por el apoyo social y el trato justo por parte de sus compañeros. 
En cuanto a la conciliación de la vida laboral y familia, la doble presencia del personal laboral el que entiende que pasa "muchas horas" en el trabajo, le dificulta compatibilizar sus tareas en determinadas épocas del curso escolar, creando un sentimiento de desasosiego.

\section{CONCLUSIÓN}

El análisis y valoración de los datos obtenidos en este estudio, aunque entendemos que no son generalizables a toda la población docente universitaria ni representativa de la misma, puede servir de base para generar un proceso de reflexión interno y externo con el resto de compañeros docentes para propiciar en los centros universitarios estudios amplios y profundos para prevenir o reducir la aparición de riesgos relacionados con la salud y el trabajo. Entendemos así, dadas las dificultades de participación del profesorado en este estudio, que la implicación activa de los trabajadores y trabajadoras es básica, si se pretende una prevención eficaz, ya que son ellos y ellas quienes van a protagonizar los cambios, que se aceptarán y entenderán mejor si han sido copartícipes de su diseño. Estos estudios de evaluación no se presentan como un fin en sí mismo, sino como un instrumento de acción preventiva y cambio, que permita identificar y localizar los riesgos de las condiciones de trabajo a la que están sometidos los trabajadores para proponer medidas resolutivas que favorezcan la salud, todos los ámbitos, de los trabajadores.

\section{REFERENCIAS BIBLIOGRÁFICAS}

Collado, S. (2008). Prevención de riesgos laborales: principios y marco normativo. Revista de Dirección y Administración de Empresas, 15, 91-117.

Comisión Europea. (1999). Guía sobre el estrés relacionado con el trabajo. ¿La sal de la vida o el beso de la muerte? Bruselas: Comisión Europea, Dirección General de Empleo y Asuntos Sociales.

Córdoba, E. (2010). Factores psicosociales y su influencia en el bienestar laboral. Buenos aires.

Departamento de Educación, Universidades e Investigación. Evaluación de factores de riesgo laboral en centros educativos. Donostia-San Sebastián.

Instituto Sindical de Trabajo Ambiente y Salud, ISTAS (2002). Manual de la versión media del método ISTAS-21 (CoPsoQ) de evaluación de riesgos psicosociales. ISTAS.

Ley 31/1995, de 8 de noviembre, de Prevención de Riesgos Laborales.

Luceño, L. y otros (2005). Evaluación de factores psicosociales en el entorno laboral. EduPsykhé, 4 (1), 19-42.

Rabadà, I. y Artazcoz, L. (2002). Identificación de los factores de riesgo laboral en docentes: un estudio Delphi. Prevención de riesgos laborales, 5 (2), 53-61. 
Sandín, B. (2002). El estrés: un análisis basado en el papel de los factores sociales. Revista Internacional de Psicología Clínica y de la Salud, 3 (1), 141-157.

Sindicato de enseñanza de Comisiones Obreras. (2006). Estudio de los factores psicosociales de riesgo en el sector de la enseñanza pública de la provincia de Málaga, a través del método Istas-21.

Vázquez, J. A. y otros. (2010). Factores psicosociales motivacionales y estado de salud. Medicina y seguridad en el trabajo, 56 (218), 12-21. 